\title{
The Dynamic Buckling of Stiffened Panels - A study using High Speed Digital Image Correlation
}

\author{
C. A. Featherston ${ }^{1, a}$, J. Mortimer ${ }^{1, b}$, M. Eaton ${ }^{1, c}$, R. L. Burguete ${ }^{2, d}$ and \\ R. Johns ${ }^{2, e}$ \\ ${ }^{1}$ Cardiff School of Engineering, Cardiff University, Queens Buildings, The Parade, Cardiff, \\ CF24 3AA, UK \\ ${ }^{2}$ Airbus UK, New Filton House, Filton, Bristol, BS99 7AR, UK \\ âFeatherstonCA@cardiff.ac.uk, ${ }^{\mathrm{b}}$ MortimerJ@cardiff.ac.uk, ${ }^{0}$ EatonM@cardiff.ac.uk, \\ ${ }^{\mathrm{d}}$ Richard.Burguete@Airbus.com, ${ }^{\mathrm{e}}$ Rhiannon.Johns@cardiff.ac.uk
}

Keywords: Dynamic buckling, stiffened panel, digital image correlation, finite element analysis.

\begin{abstract}
For a structure subjected to an intermediate velocity impact in which the duration of loading is in the order of milliseconds and in excess of the period of it's first free vibration mode there is a relationship between impact duration and buckling load. Although this relationship results in higher buckling loads for shorter duration impacts, the precise nature of the correlation depends on a number of other factors, one of which is geometry. Since the design of many lightweight structures subject to dynamic loading in this intermediate range is based on the use of a static buckling load to which a load factor is then applied, it is essential that this factor accurately represents the relationship between the two and takes of account of any variations. Failure to do so will at least result in an over designed structure and at worst in catastrophic failure.
\end{abstract}

A series of finite element analyses (FEA) have been performed in order to determine the relationship between dynamic and static buckling loads for a range of stiffened panels with differing radii of curvature.

This paper describes preliminary tests performed to determine the feasibility of using high speed digital image correlation (DIC) to study such an impact and hence provide validation of the earlier FEA analyses. These are performed on a longitudinally stiffened panel subject to uniaxial compression, clamped within a rig designed to provide built-in end conditions and allow motion of one end in the direction of loading only. The specimen is tested using an accelerated drop test rig. Impact load is monitored throughout using a load cell. Full field displacement contours are obtained using a high speed DIC system. Results are presented which demonstrate deflection contours during and after impact enabling the path of the shock wave through the specimens to be determined. An initial comparison is then made the FEA results.

\section{Introduction}

Lightweight structures consisting of thin shells reinforced with uni-directional or bi-directional stiffeners are used extensively in a range of industries including the aerospace sector. Loading of these structures under combinations of in-plane compression, bending and shear creates the potential for failure by buckling. Extensive work has therefore been carried out in order to develop guidelines for the design of a range of different panel geometries under varying boundary conditions and load cases in order to prevent this mode of failure. These include design guides such as Bruhn [1] and data sheets including those published by ESDU [2]. In each case, however loading has been assumed to be applied slowly such that a static analysis can be performed. 
In reality however, many of the loads applied to such structures for example those experienced during emergency braking and landing (Assler and Telgkamp [3])) are dynamic in nature. In such cases it has been shown that the relationship between the dynamic and static buckling loads can vary dramatically and that this variation is dependent on a number of factors. For example shorter duration impacts result in higher buckling loads (Weller et al [4]) and may be substantially in excess of the static buckling loads (Bisagni and Linde [5]) whilst longer duration impacts can result in much lower buckling loads . In order to meet future goals such as those set out by ACARE (The Advisory Council for Aeronautics Research in Europe) in their Strategic Research Agenda in Aeronautics 2020 [6] to reduce aircraft emissions by up to $80 \%$ it is essential that the former be taken into consideration to realize optimized structures and corresponding reductions in weight and therefore fuel consumption. The consequences of the latter eventuality could clearly be catastrophic.

One of the first researchers to investigate dynamic buckling was Zizicas [7] who developed a theoretical solution for the case of a simply supported rectangular plate under time-dependant inplane loads although a buckling criterion was not determined. Later Ari-Gur et al [8] showed that the dynamic buckling load of the plate was highly dependent on pulse duration and the existence of initial geometric imperfections based on experiments on a rectangular plate impacted by a mass moving in an in-plane direction. Weller et al [4] calculated the Dynamic Load Amplification Factor (DLF) for a series of beams and plates under differing pulse durations and geometric imperfection using the ADINA computer code. More recently work on stiffened panels has been performed by Bisagni and Zimmermann [9], Yaffe and Abramovich [10], Zhang et al [11] and Bisagni [12].

This paper presents research being carried out to extend the work described by examining the effects of geometry on the relationship between the static and dynamic buckling loads of a series of stiffened panels. An FEA model has been developed which has been used to study panels with differing radii of curvature. An experimental programme is now underway in order to validate this model and investigate this phenomena further. Results are presented here for a flat panel.

\section{Finite Element Analysis}

Finite element analyses have been carried out on a series of models representative of stiffened panels with radii of curvature of $400 \mathrm{~mm}, 800 \mathrm{~mm}$ and infinity (ie a flat plate) (detailed information regarding the geometry of the flat plate is given in the description of the test specimen below) in order to determine their dynamic buckling loads and relate these to their static behaviour. These were performed using the explicit solver (in which the solution is advanced kinematically from one increment to the next) available in the FEA software ABAQUS. Panels were modeled using quadrilateral S4RS elements (Shell, 4 nodes, Reduced integration, Small-strain) which are suitable for dynamic analysis of shell structures undergoing large-scale buckling behaviour with large rotations but relatively small amounts of membrane stretching and compression. In terms of boundary conditions movement in all five degrees of freedom ( 3 translations plus out-of-plane rotations) was prevented along the bottom of the panel, the sides remaining free and the top was prevented from translating in the $\mathrm{x}$ and $\mathrm{z}$ directions (Fig.3), and from rotating about the $\mathrm{x}$ and $\mathrm{y}$ axes. In each case both the plate and the stiffeners were constrained. Finally a distributed load was applied across the top edge of each of the panel, loading both plate and stiffeners according to the time profile illustrated in Fig. 1.

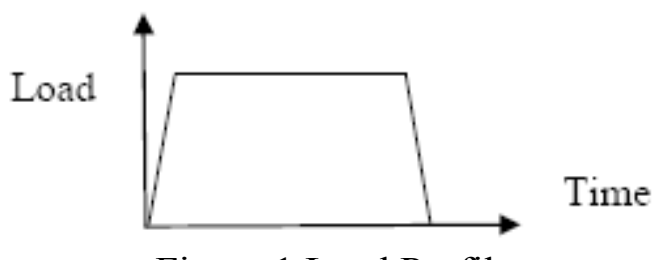

Figure 1 Load Profile 
In addition to providing specific buckling loads, results demonstrated a relationship between dynamic and static buckling loads which varied depending upon the duration of impact and the radius of curvature of the panels (Fig. 2). These indicated that for short time duration loading the dynamic buckling load is increased in comparison to its static equivalent. As load duration increases however, the critical dynamic load is seen to fall to a value considerably lower than the static one. In terms of geometry the results demonstrate an increased sensitivity to load duration in the case of a flat panel when compared to a curved one.

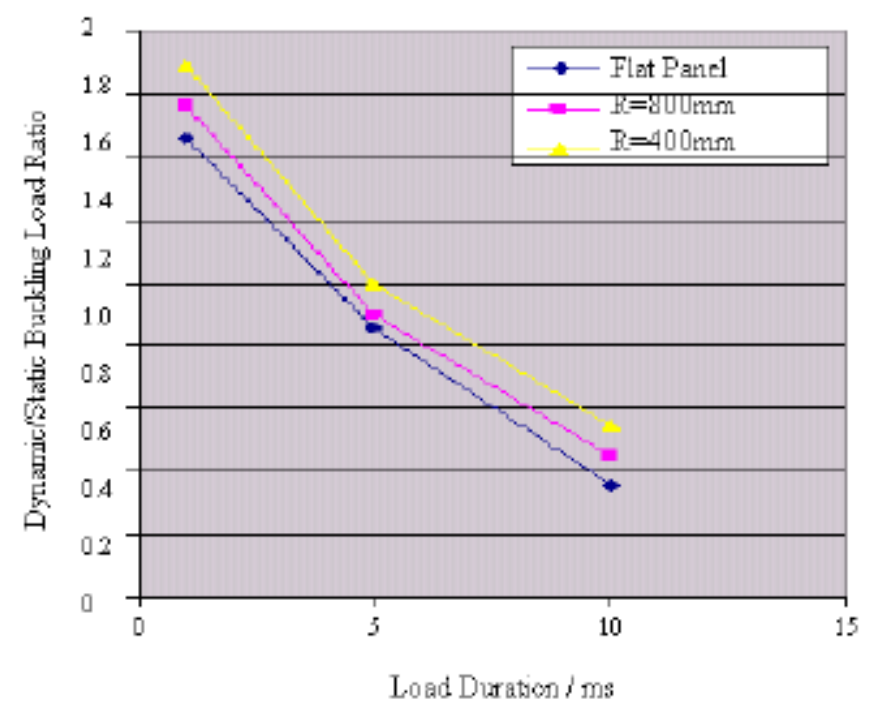

Figure 2 The Effect of Geometry on the Ratio of Dynamic to Static Buckling Load

\section{Experimental Set-Up}

Specimen. The panel tested comprised a flat aircraft grade duraluminium (BS1470 6082 - T6) plate $400 \mathrm{~mm}$ long by $400 \mathrm{~mm}$ wide and $0.5 \mathrm{~mm}$ thick to which five longitudinal blade stiffeners each $50 \mathrm{~mm}$ deep and $0.5 \mathrm{~mm}$ thick were attached using Loctite Multibond 330 and rivets shown in Fig. 3.

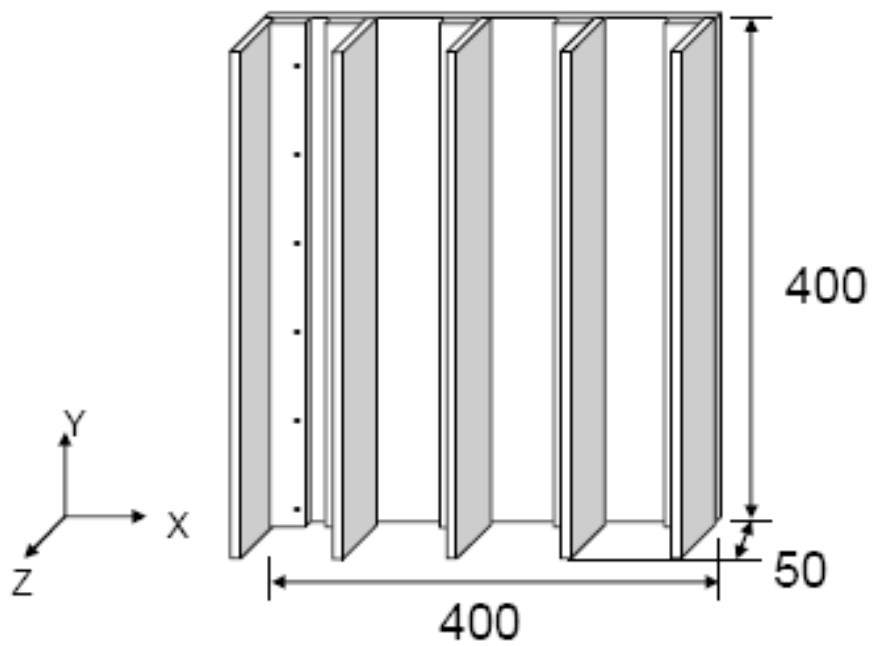

Figure 3 Panel Geometry

Test Rig. The panel was tested using the rig illustrated in Fig. 4. The loaded ends of the panel were mounted using an epoxy resin in slots machined in top and bottom plates designed to provide builtin boundary conditions. Guide rods located at the corners of the plates provided vertical alignment whilst enabling motion of the top plate in the direction of loading by means of a series of phosphor bronze bushes mounted in the upper plate. Impact loading was applied to the top plate using an 
Instron Dynatup $9250 \mathrm{HV}$ with extended width base, capable of providing impact energies of up to $1.6 \mathrm{~kJ}$ at velocities of up to $20 \mathrm{~m} / \mathrm{s}$ using an accelerated drop weight system. Tests were performed using a mass of $5.7244 \mathrm{~kg}$ dropped from $0.04,0.05$ and $0.06 \mathrm{~m}$. This generated the load profiles presented in Fig. 5, which are representative of the load duration modelled and the anticipated dynamic buckling load.
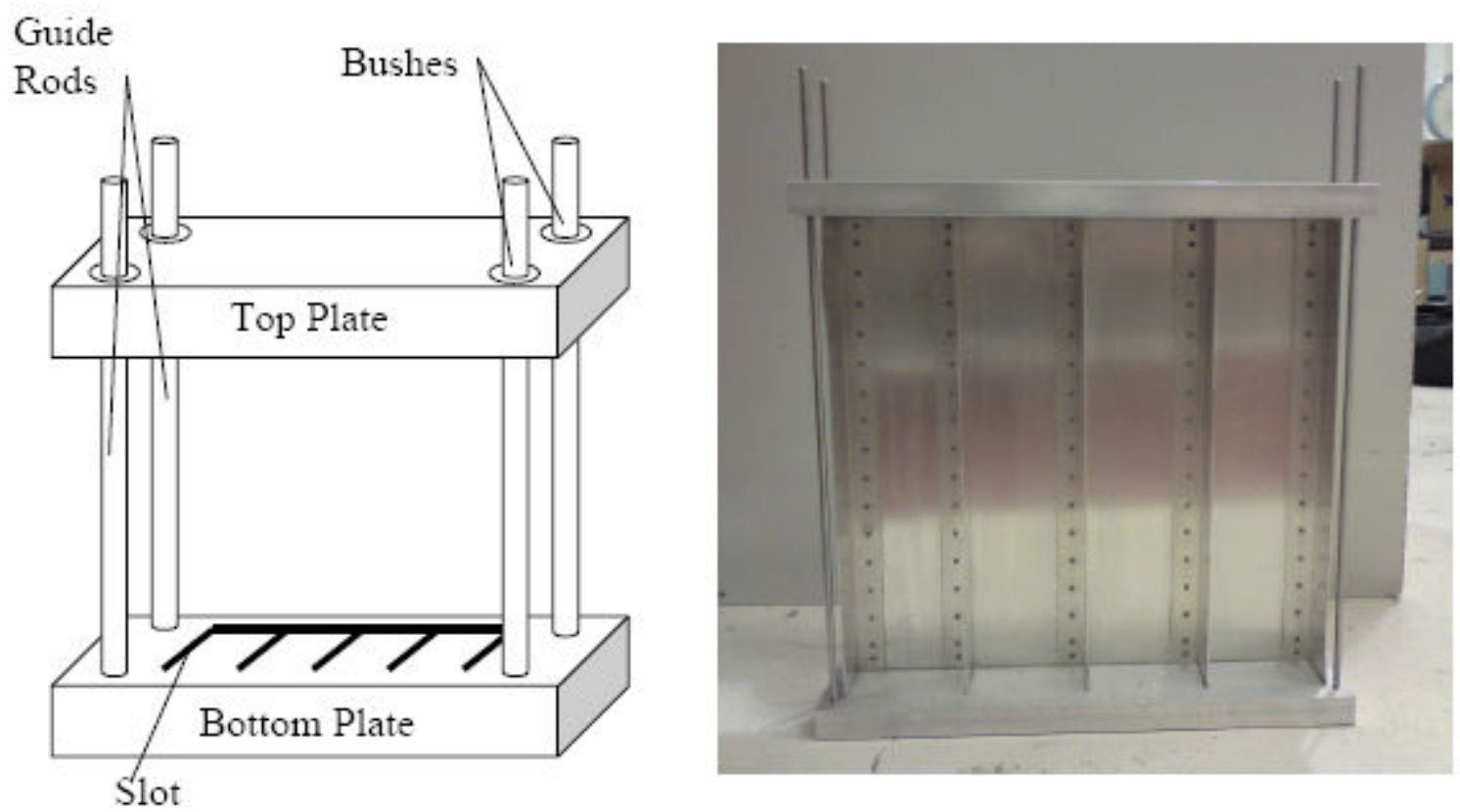

Figure 4 Test Rig.

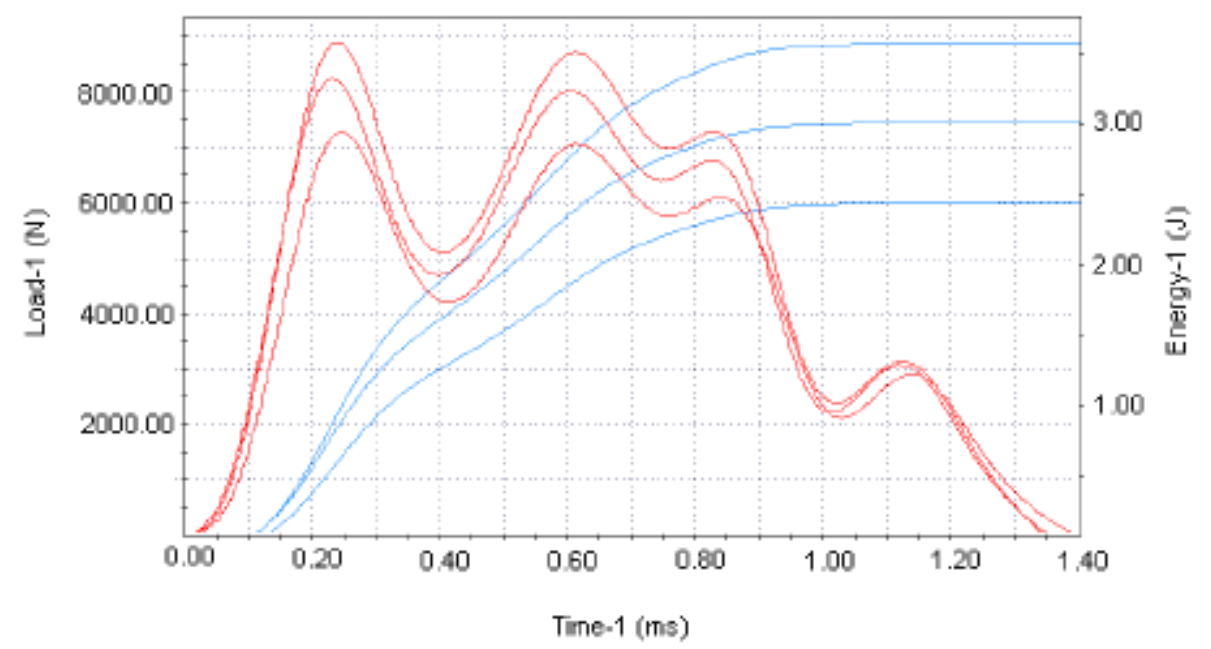

Figure 5 Load Profiles.

Digital Image Correlation. Full field displacement data was obtained for the plate only using 3D digital image correlation. This was achieved using the LIMESS system with AOS Technologies MOTIONeer high speed cameras synchronised using Correlated Solution's Vic Snap software to capture data from the panel at a resolution of $512 \times 640$ pixels and a rate of $1000 \mathrm{fps}$. This data was then post processed using Vic3D software. In addition a single camera was used to capture video footage at $10000 \mathrm{fps}$ in order to obtain qualitative data on the deformation of the stiffeners.

\section{Results}

The results obtained in terms of deformation of the panel during impact are presented in Fig. 6. Out of plane deflections can be observed near the top of each stiffener $0.1 \mathrm{~ms}$ after impact in both the 
high speed camera image (Fig 6a) and the FEA analysis (Fig 6b). These deflections are seen to move downwards through the stiffeners prior to the deformation moving into the panel (Fig 6c - 0.2, 0.3 and $1 \mathrm{~ms}$ after impact). Due to the limitations of the high speed cameras used for the DIC which could operate at a maximum of $1000 \mathrm{fps}$ for 512 × 640 pixel resolution it was not possible to obtain detailed experimental data regarding the behaviour of the back plate during impact since only one frame could be captured during the impact event. It was however possible to monitor the panel vibrations following the impact for comparison with results of the FEA. These results are provided for comparison in Fig 7. Again good correlation can be seen.
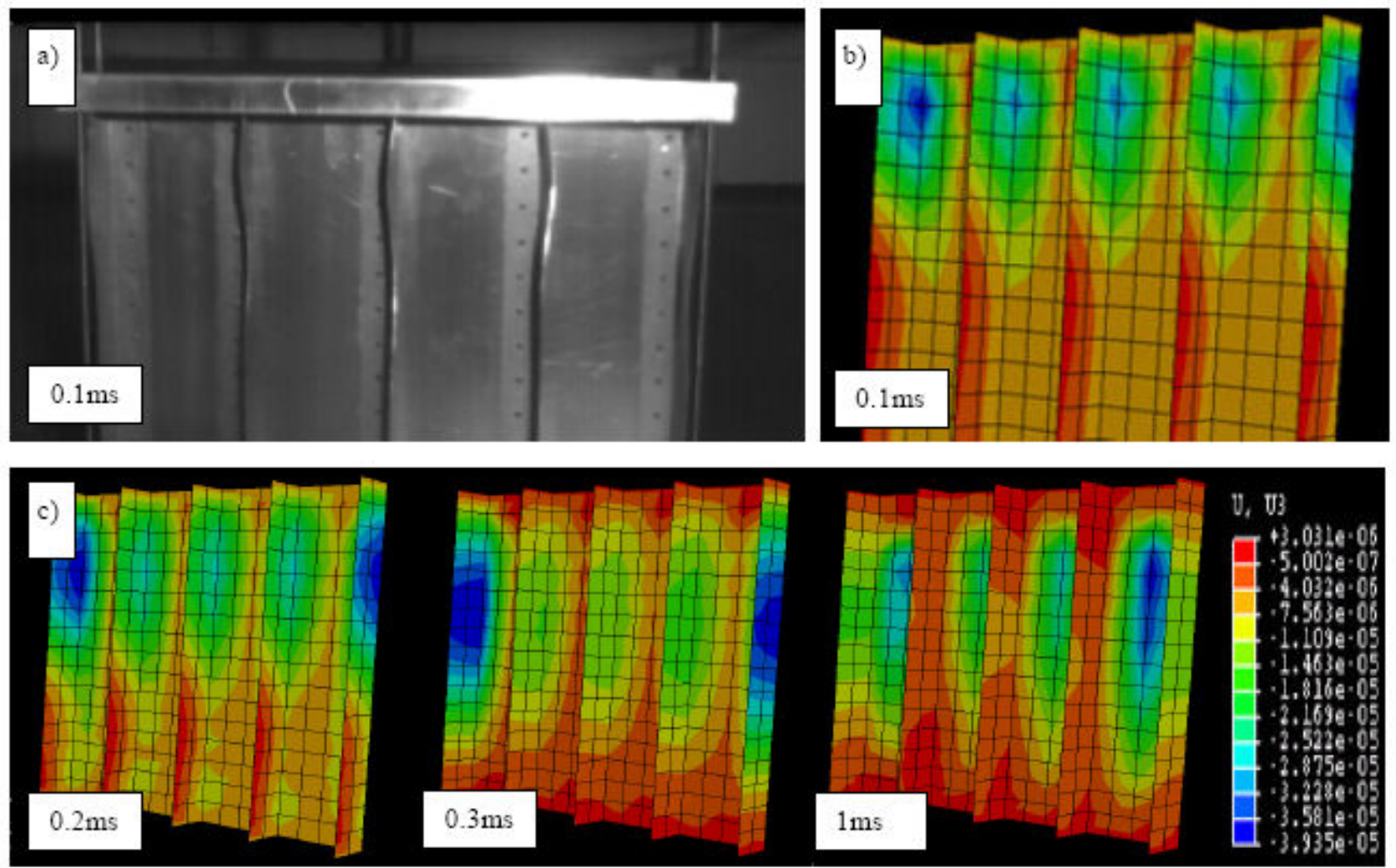

Figure 6 Out-of-Plane Displacements in the Stiffeners a), b) and Traveling Through the Panel in c).
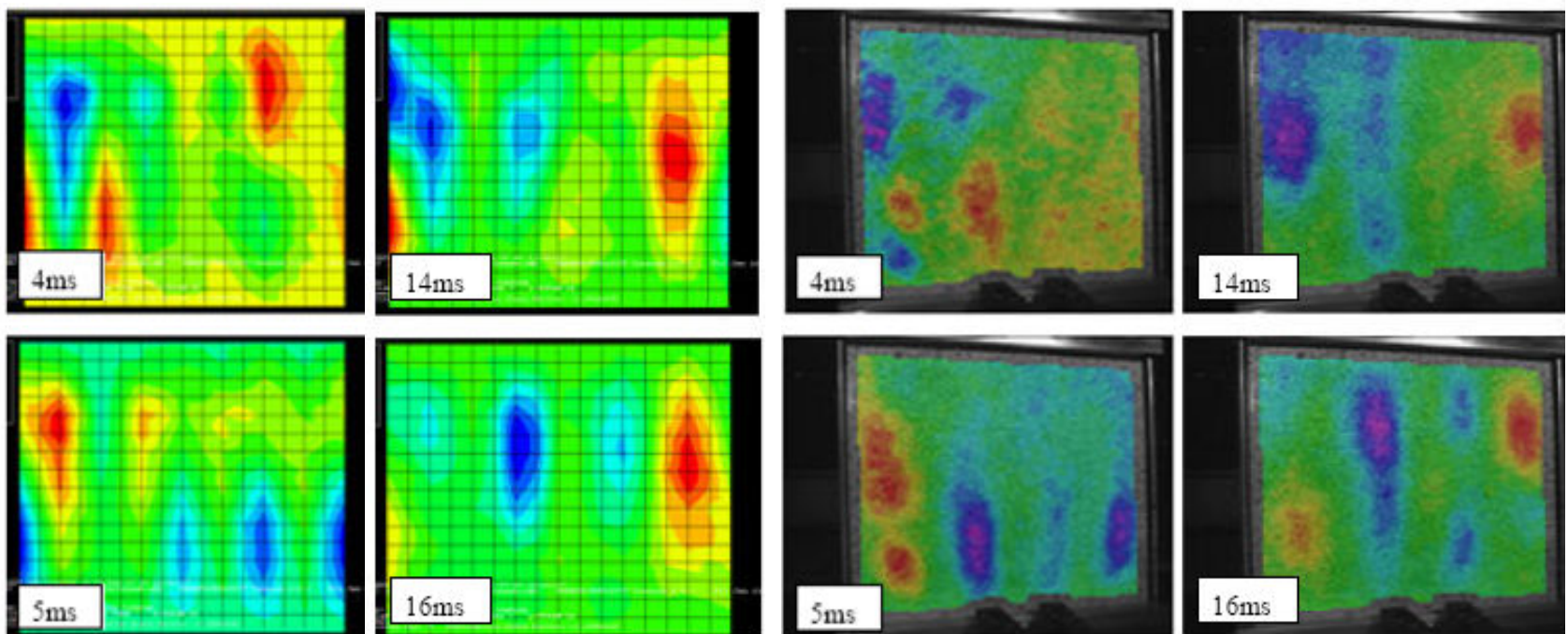

a)
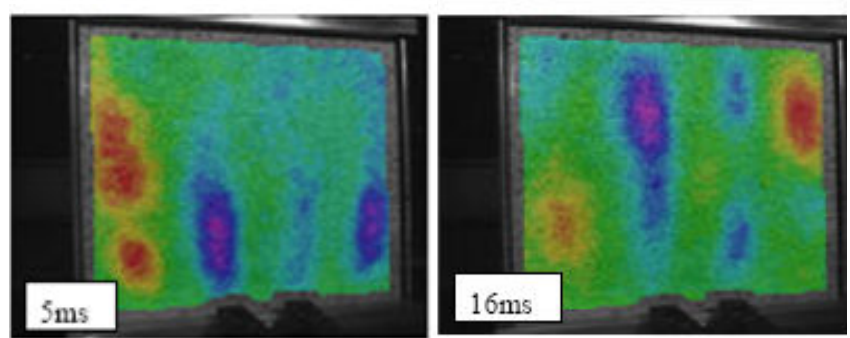

b)

Figure 7 Comparison of a) FEA and b) Experimental Out-of- Plane Vibration of the Panel Following Impact. 


\section{Conclusions}

The feasibility of using high speed photography and digital image correlation to examine the dynamic behaviour of a stiffened panel has been demonstrated. The results obtained appear to validate those previously derived using explicit finite element analyses. Further work is required to obtain more qualitative data. This will be achieved using higher speed cameras with automated synchronization to enable faster data processing.

\section{References}

[1] E.F. Bruhn: Analysis and design of flight vehicle structures. Jacobs Publishing Inc. (1973).

[2] Engineering Sciences Data Unit (ESDU): Flat Panels in Shear. Buckling of long panels with transverse stiffeners. ESDU 02.03.02 (1971).

[3] H. Assler, and J. Telgkamp: Design of aircraft structures under special consideration of NDT. 9th European Conference on NDT. Berlin (2006).

[4] T. Weller, H. Abramovich and R. Yaffe: Dynamics of beams and plates subjected to axial impact. Computers and Structures, Vol. 32(3-4) (1981), p. 835-851.

[5] C. Bisagni and P. Linde: Numerical simulation of the structural behaviour of orthotropically stiffened aircraft panels under short time duration loading. International Congress of Aeronautical Sciences. Hamburg (2006).

[6] Advisory Council for Aeronautics Research in Europe. Strategic Research Agenda in Aeronautics 2020 (2004).

[7] G.A. Zizicas: Dynamic buckling of thin plates. Trans. ASME, 74(7) (1952), p. 12571268.

[8] J. Ari-Gur, J. Singer and T. Weller: Dynamic buckling of plates under longitudinal impact. Israel J Techno, Vol. 19 (1981), p. 57-64.

[9] C. Bisagni and R. Zimmerman, Buckling of axially compressed fiber composite cylindrical shells due to impulsive loading. Proceedings of the European Conference on Spacecraft Structures, Materials and Mechanical Testing (1998), p. 557-562.

[10] R. Yaffe and H. Abromovich: Dynamic buckling of cylindrical stringer stiffened shells. Computers and Structures, Vol. 81 (2003), p. 1031-1039.

[11] T. Zhang, T. Liu, Y. Zhao and J. Luo: Nonlinear dynamic buckling of stiffened plates under inplane impact loading. Journal of Zhejiang University of Science, Vol. 5(5) (2004), p. 609617.

[12] C. Bisagni, Dynamic buckling test of cylindrical shells in composite materials. $24^{\text {th }}$ International Congress of the Aeronautical Sciences, Yokahama (2004). 